Journal of

Synchrotron

Radiation

ISSN 0909-0495

Received 25 April 2004

Accepted 5 January 2005

\section{Beam-size effects in radiation damage in insulin and thaumatin crystals}

\author{
C. Schulze-Briese,* A. Wagner, T. Tomizaki and M. Oetiker
}

Swiss Light Source at Paul Scherrer Institute, CH-5232 Villigen, Switzerland. E-mail: clemens.schulze@psi.ch

\begin{abstract}
Cryocooled insulin and thaumatin crystals were irradiated in a series of alternating data collections and high-dose-rate exposures using either a vertically focused or vertically defocused beam. The main result is that the radiation damage is limited to the exposed region, which can be explained by the short range of the photoelectrons and the Auger electron cascade produced by light elements. Consequently, the unexposed angular range provides significantly improved data quality and electron density compared with previously exposed angular wedges of the crystal when a vertically focused beam is used, while no differences are observed between a fresh wedge and an exposed region for the vertically defocused beam. On the other hand, the focused beam provides higher $I / \sigma_{I}$ ratios at high resolution than homogeneous sample illumination but also causes more rapid sample deterioration.
\end{abstract}

(C) 2005 International Union of Crystallography Printed in Great Britain - all rights reserved

\section{Introduction}

After initial crystal quality, radiation damage is the most important limitation for successful structure solution at thirdgeneration synchrotron sources. However, even when the structures can be solved, they may be altered by radiation damage (decarboxylations of acidic residues and disulfide bond breakage); the active sites of enzymes are particularly susceptible to radiation damage (Burmeister, 2000), and metalloenzymes may be reduced by radiation. Both effects may lead to misinterpretations of structural features. So far, He cooling or the use of radical scavengers has not proven to be useful as a general means of controlling radiation damage (Garman \& Nave, 2002). On the other hand, the achievable focal spot sizes at low-emittance third-generation synchrotron sources are becoming smaller, thereby delivering even higher flux densities, and several new beamlines will provide the possibility to focus the beam to a size of $20 \mu \mathrm{m}$ or below (A. Thompson \& L. Duke, personal communication). At the high-resolution diffractometer of the SLS protein crystallography beamline X06SA, the beam can be focused in the vertical direction to $7 \mu \mathrm{m}$ (Rossetti et al., 2002), owing to the small size of the electron beam in the storage ring and to the outstanding mirror quality (Carl Zeiss Synchrotron Optics). This small beam size results in a reduction of the background and a small beam on the detector, leading to a higher-resolution diffraction limit. However, the question arises as to whether the higher flux density gives rise to enhanced radiation damage even for relatively large samples $(>100 \mu \mathrm{m})$. While it is common practice to translate samples that are larger than the beam, little is known about the effect that the rotation of the sample has on the radiation damage to crystals whose dimension in the direction perpendicular to the rotation axis exceeds that of the beam. The experiments described below address this question by studying the radiation damage to insulin and thaumatin crystals caused by beams of different vertical size but with the same total flux. Minimization of the effects of radiation damage on the data quality by means of an optimal beam geometry and crystal translation strategy is particularly important for S-SAD experiments. These experiments often require the collection of significantly more than $180^{\circ}$ of data in order to obtain a sufficient data redundancy, and they are carried out at long wavelengths where careful flux adjustment is required to compensate for the increase of the photoelectric cross section, which would otherwise entail a higher dose to the crystal thereby causing more rapid sample deterioration for the same incident flux. Another way to reduce radiation damage and improve data quality in mediumresolution data collections, which will not be discussed here, is to focus the beam on the detector, whereby the flux density will be reduced on the sample but maximized on the detector (Wikoff et al., 2000).

\section{Materials and methods}

Cubic porcine $\mathrm{Zn}$-free insulin (I5523, Fluka) was dissolved in $0.2 M \mathrm{Na}_{3} \mathrm{PO}_{4} / \mathrm{Na}_{2} \mathrm{HPO}_{4}$ buffer (pH 11) containing $0.01 \mathrm{M}$ $\mathrm{Na}_{3}$ EDTA. Crystals (space group $12{ }_{1} 3,79.1 \AA$, $5766 \mathrm{D}, 65 \%$ solvent fraction) were grown from $25 \%$ ethylene glycol at $\mathrm{pH} 9.5$ by means of the hanging-drop method. No further addition of cryoprotectant was necessary. Crystals of tetragonal thaumatin (T7638, Fluka) were obtained from a $2 \mathrm{MNa-}$ 
Table 1

Properties of the vertically focused and defocused beams during the experiments.

The beam dimensions refer to the FWHM of a Gaussian-shaped beam profile of the focused beam and to that of the flat-topped profile of the defocused beam in the vertical direction. The photon energy was $8 \mathrm{keV}$ and the detector distance was $65 \mathrm{~mm}$. The flux was measured by means of a $12 \mu \mathrm{m}$-thick Si-PIN diode (www.sintef.no), corrected for air and entrance-window absorption. Dose rates were calculated by means of the program RADDOSE (Murray et al., 2004).

\begin{tabular}{|c|c|c|c|c|c|}
\hline & Insulin I & Insulin II & Insulin III & Thaumatin I & Thaumatin II \\
\hline Crystal size $(\mu \mathrm{m})$ & $180 \times 180 \times 160$ & $130 \times 130 \times 100$ & $125 \times 125 \times 80$ & $300 \times 150 \times 100$ & $350 \times 150 \times 150$ \\
\hline Beam size $(\mathrm{h} \times \mathrm{v})(\mu \mathrm{m})$ & $82 \times 10$ & $85 \times 170$ & $82 \times 10$ & $82 \times 10$ & $85 \times 170$ \\
\hline Flux (photons s ${ }^{-1}$ ) & $1.25 \times 10^{12}$ & $1.25 \times 10^{12}$ & $0.75 \times 10^{12}$ & $0.75 \times 10^{12}$ & $0.75 \times 10^{12}$ \\
\hline Flux density (photons $\mathrm{s}^{-1} \mathrm{~mm}^{-2}$ ) & $1.5 \times 10^{15}$ & $8.5 \times 10^{13}$ & $0.9 \times 10^{15}$ & $0.9 \times 10^{15}$ & $0.9 \times 10^{15}$ \\
\hline Dose rate $\left(\mathrm{MGy} \mathrm{s}^{-1}\right)$ & 1.9 & 0.11 & 1.1 & 1.1 & 0.065 \\
\hline Dose of attenuated beam $\left(\mathrm{kGy} \mathrm{s}^{-1}\right)$ & 60.4 & 3.4 & 9.1 & 9.1 & 0.52 \\
\hline
\end{tabular}

Table 2

Exposure conditions for insulin I, insulin II and insulin III.

The dose (columns 6,8 and 9) corresponds to the total accumulated dose at the end of the exposures without taking sample rotation into account, whereas the 'volume-averaged' dose (column 7) takes into account the swept volume. The thickness of the Al filter (column 5) during data collection of insulin III was $350 \mu \mathrm{m}$.

\begin{tabular}{|c|c|c|c|c|c|c|c|c|}
\hline \multirow[b]{2}{*}{ Name } & \multirow[b]{2}{*}{$\varphi$ range $\left(^{\circ}\right)$} & \multirow[b]{2}{*}{$\Delta \varphi\left(^{\circ}\right)$} & \multirow[b]{2}{*}{$T(\mathrm{~s})$} & \multirow[b]{2}{*}{$\mathrm{Al}(\mu \mathrm{m})$} & \multicolumn{4}{|l|}{ Dose } \\
\hline & & & & & $\begin{array}{l}\text { Insulin I } \\
\text { (MGy) }\end{array}$ & $\begin{array}{l}\text { Insulin I } \\
\text { (volume-averaged) } \\
\text { (MGy) }\end{array}$ & $\begin{array}{l}\text { Insulin II } \\
\text { (MGy) }\end{array}$ & $\begin{array}{l}\text { Insulin III } \\
\text { (MGy) }\end{array}$ \\
\hline Reference & $0-60$ & 1 & 1 & 250 & 3.6 & 0.6 & 0.2 & 0.5 \\
\hline Irradiation-1 & $0-60$ & 60 & 10 & - & 22.6 & 4.0 & 1.3 & 12.0 \\
\hline Rad-1 & $0-60$ & 1 & 1 & 250 & 26.2 & 4.6 & 1.5 & 12.5 \\
\hline Irradiation-2 & $0-60$ & 60 & 10 & - & 45.2 & 8.0 & 2.6 & 23.9 \\
\hline Rad-3 & $0-60$ & 1 & 1 & 250 & 109.4 & 19.4 & 6.2 & 59.2 \\
\hline Irradiation-4 & $0-60$ & 60 & 30 & - & 166.4 & 29.5 & 9.4 & 93.4 \\
\hline Rad-4 & $0-60$ & 1 & 1 & 250 & 170.0 & 30.1 & 9.7 & 93.9 \\
\hline Fresh & $90-150$ & 1 & 1 & 250 & 3.6 & & 9.9 & \\
\hline Heat-1 & $90-150$ & 1 & 1 & 250 & 7.2 & & 10.1 & N/A \\
\hline Heat-2 & $0-60$ & 1 & 1 & 250 & 173.6 & & 10.3 & N/A \\
\hline
\end{tabular}

K-tartrate, $1 M$ MES solution (space group $P 4_{1} 21_{1} 2,58.5 \AA$, $151.35 \AA^{3}, 22188 \mathrm{D}$, solvent fraction $43 \%$ ) by means of the hanging-drop method. For cryoprotection the crystals were soaked in $20 \%$ glycerol for $10 \mathrm{~min}$.

Two experiments were carried out during two runs. In all experiments the beam was horizontally focused on the sample to a Gaussian beam profile of $82-85 \mu \mathrm{m}$ full width at halfmaximum. The focused beam has an elliptical shape and a Gaussian beam profile in the horizontal and vertical directions, while vertical defocusing leads to a flat-topped vertical profile (see Table 1). Two insulin and two thaumatin crystals were irradiated with vertically focused and defocused beams, respectively. They will be referred to in the following as 'insulin I', 'insulin II', 'thaumatin I' and 'thaumatin II'. In a control experiment, a third insulin crystal was irradiated with the focused beam, referred to hereafter as 'insulin III'. The protocol was identical for all crystals and the beam parameters and crystal sizes are summarized in Table 1 . The first crystal size value refers to the dimension parallel to the horizontal spindle axis. Crystals were not translated during the course of the experiment. All data sets consist of 60 images, each of $1^{\circ}$ oscillation with $1 \mathrm{~s}$ exposure time, collected at a detector distance of $65 \mathrm{~mm}$ and a beam energy of $8 \mathrm{keV}$. The storagering beam current was $300 \mathrm{~mA}$ throughout the experiments owing to top-up operation of the ring. The primary beam was attenuated with $250 \mu \mathrm{m}$ of $\mathrm{Al}$ for insulin I and II and $350 \mu \mathrm{m}$ for insulin III and for thaumatin I and II, in order to minimize the number of overloads at an exposure time of $1 \mathrm{~s}$ (see Table 2). After collection of a reference data set ('ref'), the crystals were exposed twice for $10 \mathrm{~s}$ with the unattenuated beam covering the same $60^{\circ}$ wedge and twice for $30 \mathrm{~s}$. After each irradiation, a data set was collected with an attenuated beam (data sets 'rad-1'-'rad-4'). Finally the crystals were rotated by $90^{\circ}$ and another data set was taken in this previously unexposed region (data set 'fresh'). In addition, insulin I and insulin II crystals were warmed to $180 \mathrm{~K}$ at a rate of $10 \mathrm{~K} \mathrm{~min}^{-1}$ in an attempt to visualize the radiation damage and to investigate the effect of enhanced diffusion of free radicals. Data sets taken at $100 \mathrm{~K}$ (cooling rate $3 \mathrm{~K} \mathrm{~min}^{-1}$ ) after the temperature treatment are referred as 'heat-1' and 'heat-2'. Table 2 summarizes the exposure conditions for insulin I and II. The doses given in the table refer to the dose to a stationary crystal and do not take the sample rotation and the associated increase of the exposed sample volume into account. When the effective sample volume owing to sample rotation is used for the calculation of the average dose, the numbers decrease significantly as summarized in Table 2 for insulin I and in Table 3 for thaumatin I. It is important to note that the dose distribution within the crystal is by no means homogeneous when the beam is significantly smaller than the sample, as illustrated in Fig. 1 for insulin I.

All data sets were processed using $X D S$ and scaled using XSCALE (Kabsch, 1993). For the reference data sets, structure models from S-SAD experiments (A. Wagner, unpub- 
Table 3

Exposure conditions for thaumatin I and thaumatin II.

\begin{tabular}{|c|c|c|c|c|c|c|c|}
\hline \multirow[b]{2}{*}{ Name } & \multirow[b]{2}{*}{$\varphi$ range $\left(^{\circ}\right)$} & \multirow[b]{2}{*}{$\Delta \varphi\left(^{\circ}\right)$} & \multirow[b]{2}{*}{$T(\mathrm{~s})$} & \multirow[b]{2}{*}{$\mathrm{Al}(\mu \mathrm{m})$} & \multicolumn{3}{|l|}{ Dose } \\
\hline & & & & & $\begin{array}{l}\text { Thaumatin I } \\
\text { (MGy) }\end{array}$ & $\begin{array}{l}\text { Thaumatin I } \\
\text { (volume-averaged) } \\
\text { (MGy) }\end{array}$ & $\begin{array}{l}\text { Thaumatin II } \\
\text { (MGy) }\end{array}$ \\
\hline Reference & $0-60$ & 1 & 1 & 350 & 0.5 & 0.1 & 0.1 \\
\hline Irradiation-1 & $0-60$ & 60 & 10 & - & 12.0 & & 2.3 \\
\hline Rad-1 & $0-60$ & 1 & 1 & 350 & 12.5 & 3.1 & 2.4 \\
\hline Irradiation-2 & $0-60$ & 60 & 10 & - & 23.9 & & 4.6 \\
\hline Rad-3 & $0-60$ & 1 & 1 & 350 & 59.2 & 14.8 & 11.4 \\
\hline Irradiation-4 & $0-60$ & 60 & 30 & - & 93.4 & & 18.0 \\
\hline Rad-4 & $0-60$ & 1 & 1 & 350 & 93.9 & 23.5 & 18.1 \\
\hline Fresh & 90-150 & 1 & 1 & 350 & & & \\
\hline
\end{tabular}

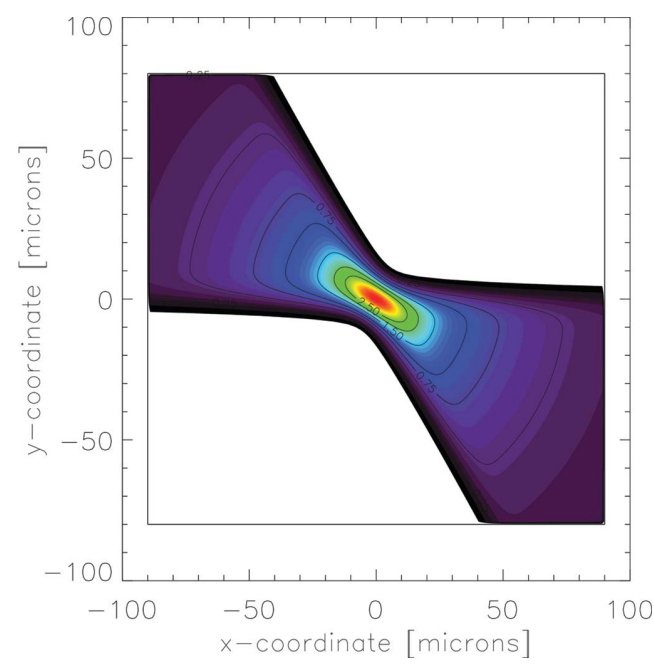

Figure 1

Dose distribution for insulin I after collection of the reference data set. The beam has a Gaussian profile with a full width at half-maximum of $10 \mu \mathrm{m}$. The square indicates the crystal dimensions. The attenuation of the beam within the crystal has not been taken into account. The colours represent low ( $0.25 \mathrm{MGy}$, black) to high (3 MGy, red) dose. The white area is the unexposed crystal area.

lished results) were used for initial rigid-body refinement using SHELXL (Sheldrick \& Schneider, 1997). In the subsequent isotropic refinements with $S H E L X L$, a water-solvent model was established from the highest peaks in the difference Fourier analysis. They were then used as initial models for the refinement of data sets rad-1-rad-4 and fresh. After a rigidbody refinement, isotropic refinements were performed using SHELXL for each data set. In order to prevent model bias and to obtain a qualitative picture of the radiation damage in the disulfide bonds, the same refinement procedure was repeated starting with models without sulfur atoms.

\section{Results and discussion}

\subsection{Non-specific damage}

Several parameters are frequently used as an indicator for radiation damage during measurements at synchrotron beamlines. Beside an increase of Wilson B factors, the unit-cell

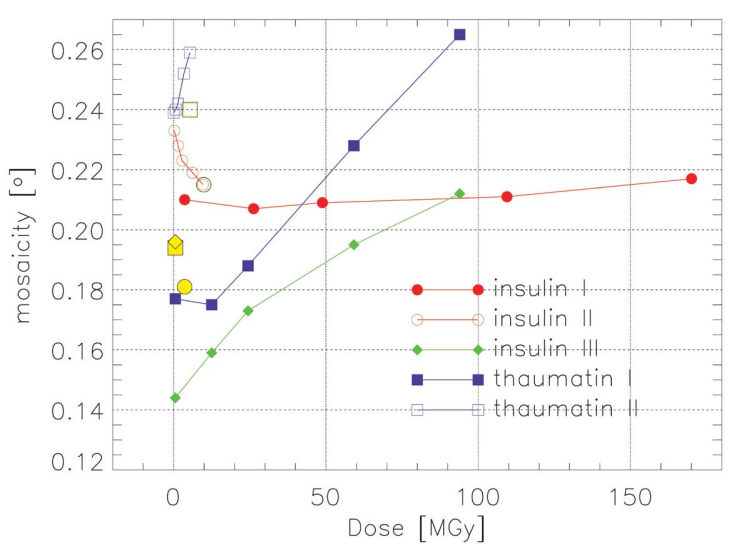

Figure 2

Change of the mosaicity as a function of the dose. The larger yellow symbols represent the data sets fresh for each crystal.

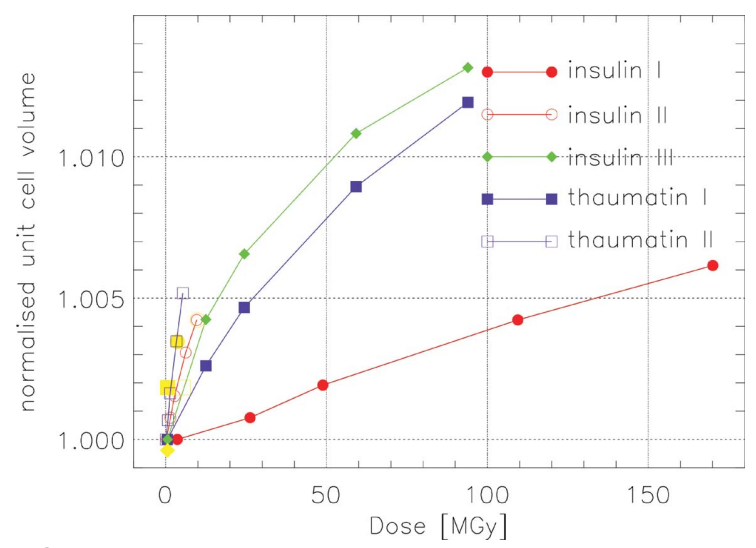

Figure 3

Unit-cell expansion as a function of the dose. The larger yellow symbols represent the data sets fresh for each crystal.

volume, internal $R$-factors and crystal mosaicity (Ravelli \& McSweeney, 2000; Ravelli et al., 2002) can serve as criteria to stop data acquisition or to translate the sample. Fig. 2 summarizes the change of the mosaicity (as output by $X D S$ ) as a function of the dose to the crystals. While a slight increase could be observed in the case of the focused beam for insulin I, the increase is significantly larger in the case of insulin III, and even bigger for thaumatin I. More surprisingly, the mosaicity of the insulin crystal decreased when the beam was 
defocused but increased for thaumatin II. In all cases the freshly exposed crystal region at $90^{\circ}$ showed significantly reduced mosaicity as compared with values observed in rad-4, both for the vertically focused and for the vertically defocused beam. Since the change in the crystal mosaicity was different for all crystals and, more importantly, since no systematic change of the mosaicity was observed during the individual data sets, mosaicity does not seem to be a good indicator of radiation damage.

Fig. 3 shows the unit-cell volume normalized to the value of the reference data set as a function of dose. A significant increase can be observed for all crystals. The total unit-cell expansion is of similar magnitude for insulin I and II despite the different dose rates. The unit-cell expansion of thaumatin I and insulin III clearly displays a non-linear dose dependence, similar to the observation by Teng \& Moffat (2000) for tetragonal hen egg-white lysozyme. They concluded that the non-linear effect above the threshold of $10 \mathrm{MGy}$ was due to secondary and maybe tertiary radiation damage effects. However, in the present study, all crystals except insulin I exhibit a non-linear behaviour even below $10 \mathrm{MGy}$. On the other hand, the initial slope of insulin III is almost 12 times larger than that of insulin I, which shows a linear dependency of the unit-cell expansion well beyond the Henderson limit of $20 \mathrm{MGy}$ (Henderson, 1990). These results corroborate the conclusions of Murray \& Garman (2002), that the unit-cell expansion is highly variable for different crystals of the same protein. The unit-cell volumes of the freshly exposed angular wedges do not show a consistent behaviour, which can most likely be attributed to the different anisotropies of the crystals. Finally, no unit-cell change was observed during individual data collections.

The decrease of the $I / \sigma_{I}$ ratio at high resolution is a more sensitive indicator for radiation damage than the parameters already discussed. Fig. 4 shows the $I / \sigma_{I}$ ratios of the reference data sets of insulin as a function of the resolution for the focused and the vertically defocused beam, as well as the curves for the different data sets normalized to the respective reference data set for that crystal. While the freshly exposed volume reaches the reference value in the case of the focused beam, the $I / \sigma_{I}$ ratio continuous to deteriorate when the beam is vertically defocused. However, the normalized $I / \sigma_{I}$ ratios decrease more rapidly with dose for the focused beam than for the defocused beam. It is interesting to note that the strong decrease of $I / \sigma_{I}$ for the cryocooled samples is mainly limited to the high-resolution shells, while Blake \& Phillips (1962) found a significant reduction of the intensities even at low resolution for sperm-whale myoglobin crystals mounted in capillaries at room temperature already at a dose level of $0.05-0.5 \mathrm{MGy}$. The authors interpreted the loss of diffracting power over the whole range of resolution as a signature of the formation of an amorphous component in the crystal, in contrast to a highly disordered part, which still gives rise to diffraction at low resolution.

Finally, comparison of the reference curves also shows that the focused beam results in a better signal-to-noise ratio at high resolution despite the smaller values at low resolution.
The analysis of the internal $R$-factors as a function of resolution gave a similar result, which is summarized in Fig. 5 for insulin and thaumatin. Comparison of the reference data sets shows that the focused beam gives better data statistics at high resolution than the defocused beam. For insulin $\mathrm{I}$, the $R$ factor for the freshly exposed crystal volume was almost identical to that of the reference over the whole range of resolution (Fig. 5a) while, for data set fresh of insulin II, no recovery of the data quality was observed (Fig. $5 b$ ). On the other hand it is worth noticing that the increase of the $R$-factor at high resolution is more marked for the focused beam. The results shown for thaumatin in Figs. 5(c) and 5(d) show the same overall trend for the focused and defocused beam. However, the effect of the recovery of the internal $R$-factor observed for data set fresh of thaumatin I is less pronounced than for insulin I. On the other hand a slight improvement of $R_{\text {int }}$ is observed for data set fresh of thaumatin II. Since this improvement is accompanied by a recovery of the mosaicity to the value for data set ref, and of the unit-cell volume to the value for data set rad-2, it is likely that these improvements are at least in part caused by crystal anisotropy. The control experiment insulin III gave similar results for $R_{\mathrm{int}}$ and $I / \sigma_{I}$ as insulin I, in particular with respect to the recovery of the crystal quality for the data set fresh.

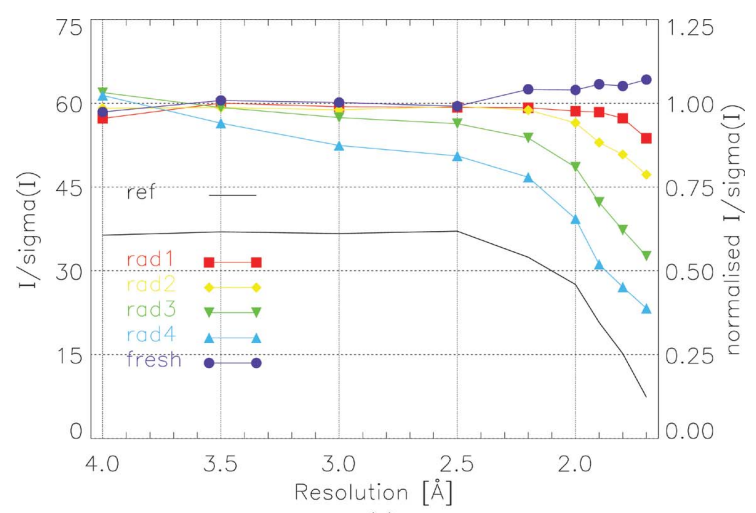

(a)

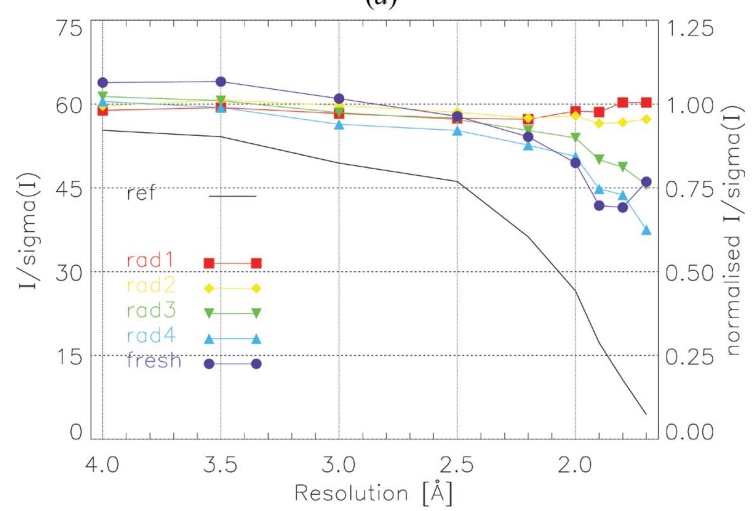

(b)

Figure 4

$I / \sigma_{I}$ of the reference data set (ref) as a function of the resolution. The $I / \sigma_{I}$ values of the other data sets are normalized to those of the reference data set at the corresponding resolution. (a) Insulin I (vertically focused beam), (b) insulin II (vertically defocused beam). 


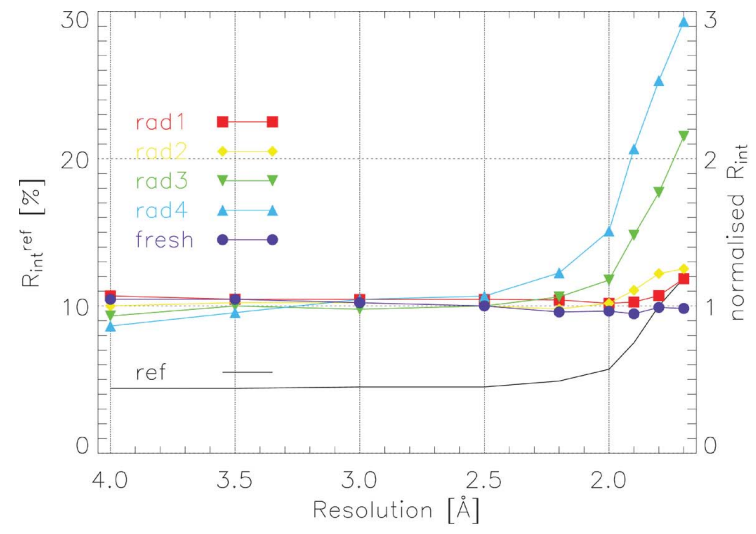

(a)

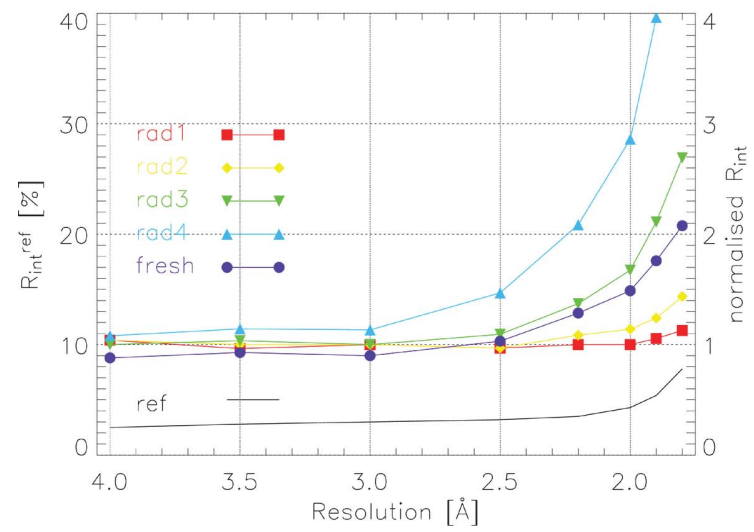

(c)

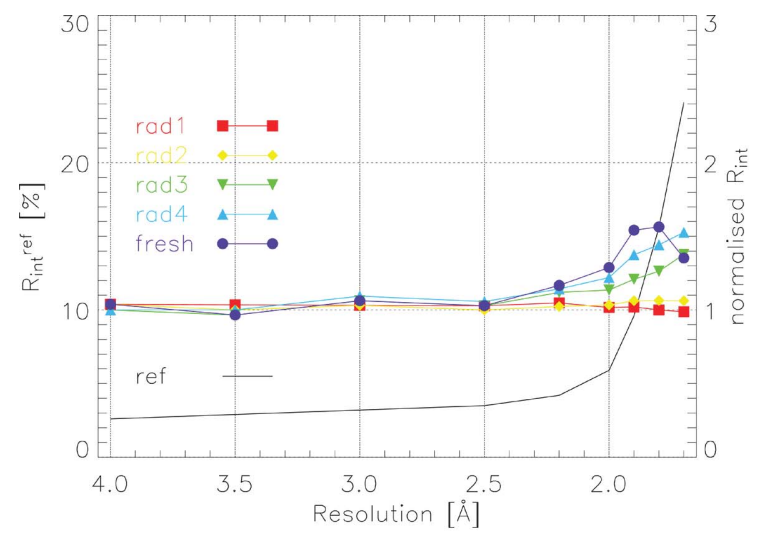

(b)

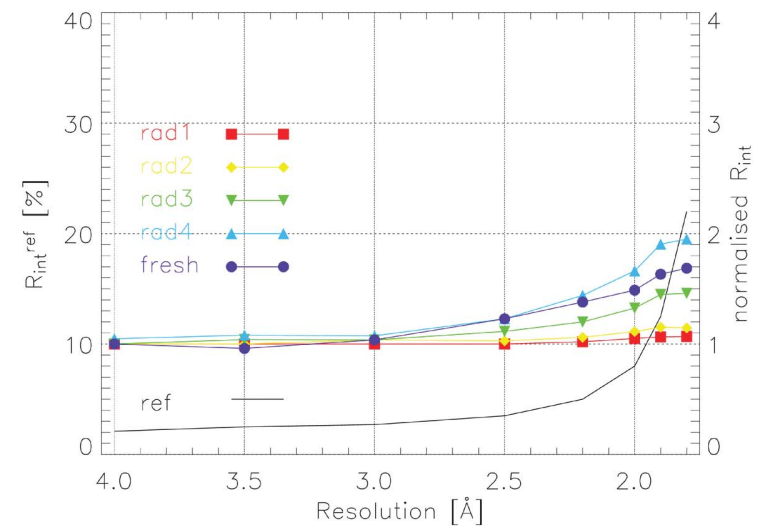

(d)

Figure 5

$R_{\text {int }}$ of the reference data set (ref) as a function of the resolution. Same data normalization as in Fig. 4. (a) Insulin I, (b) insulin II, (c) thaumatin I (vertically focused beam), $(d)$ thaumatin II (vertically defocused beam).

\subsection{Specific damage}

In order to investigate whether the behaviour of the global parameters is also reflected in the electron densities, the data sets were refined as described above and the disulphide bonds inspected [see Burmeister (2000) for a discussion of the radical reaction involved in the cleavage of disulfide bonds]. Whereas two of the three disulfide bonds in insulin do not show a significant change after irradiation, the one formed by cysteines A7 and B7 was found to be highly sensitive to radiation. Fig. 6 shows the $F_{o}-F_{c}$ maps for the focused and defocused beam. The general trend is an asymmetric decrease of the electron density. This asymmetry in the loss of electron density of the cystines has previously been observed for winged bean chymotrypsin inhibitor (Ravelli \& McSweeney, 2000). The electron density vanishes much more quickly in the case of the focused beam than for the defocused beam for the equivalent data sets, whereas the loss of density is similar or even smaller when data sets with the same average dose (see Tables 2 and 3) are compared, e.g. data set rad-2 of insulin I with an average dose of $8.6 \mathrm{MGy}$ with data set rad-3 of insulin II where the dose amounted to $6.2 \mathrm{MGy}$. This means that the dose calculated for a stationary crystal in Grays $(1 \mathrm{~Gy}=$ $1 \mathrm{~J} \mathrm{~kg}^{-1}$ ), as given by

$$
D=\left(\frac{\mu}{\rho}\right) \frac{n T e E}{A} \times 10^{11}
$$

with $\mu / \rho\left(\mathrm{cm}^{2} \mathrm{~g}^{-1}\right)$ denoting the mass absorption coefficient of the crystal, $n$ the number of incident photons $\mathrm{s}^{-1}, T$ the exposure time (s), $E$ the photon energy $(\mathrm{eV}), A$ the target area $\left(\mu \mathrm{m}^{2}\right)$ and $e=1.6 \times 10^{-19} \mathrm{~J} \mathrm{eV}^{-1}$ (O'Neill et al., 2002), has to be used with caution to estimate possible radiation damage. A three-dimensional model that takes beam and crystal dimensions into account is more appropriate to this end. In the case of the focused beam, the electron density of the data set taken at $90^{\circ}$ is equivalent to that of the reference data set, while there is no improvement observed in the case of the defocused beam. This means that there is good correspondence between the global parameters like the internal $R$-factor and the $I / \sigma_{I}$ ratio and the specific radiation damage as it becomes manifest in a loss of electron density. More importantly, the damage to the crystal is confined to the irradiated angular wedge only. Finally, in both cases there is a severe loss of electron density of the disulfide bridges well below the Henderson limit of 20 MGy (Henderson, 1990).

Fig. 7 shows the electron density of the disulfide bond Cys159-Cys164 of a thaumatin crystal that had been exposed to a focused beam (data set thaumatin I). Cys159 clearly shows two distinct conformations in the reference data set, which disappear after irradiation (rad-4). When a fresh angular wedge of the crystal is exposed, the disorder can be observed again, although the second conformation is at slightly lower occupancy. This implies that for thaumatin the radiation 

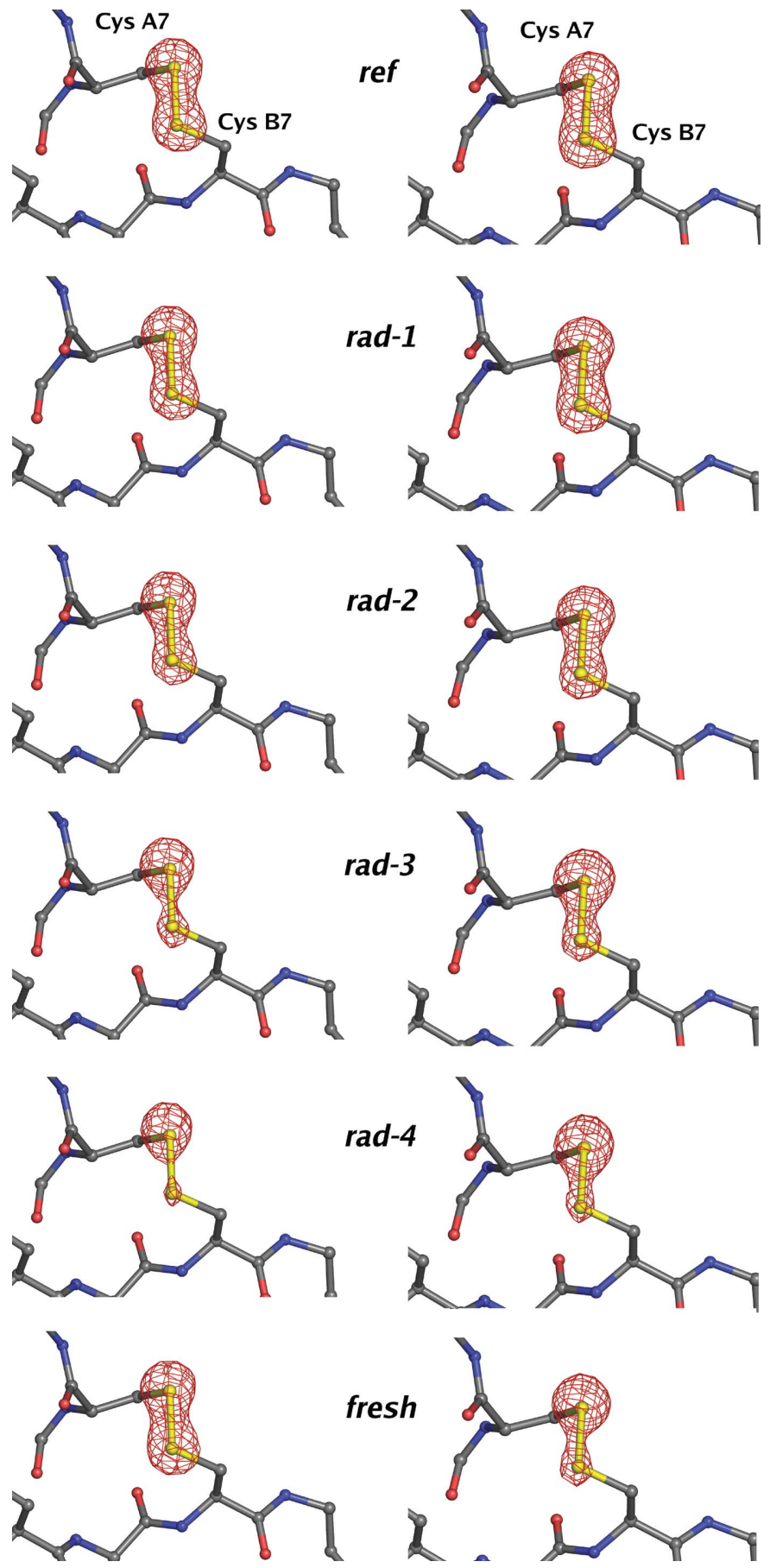

Figure 6

$F_{o}-F_{c}$ difference Fourier maps contoured at $5 \sigma$ showing the evolution of radiation damage in the disulfide bridge between cysteines A7 and B7 together with the model from the reference data set for insulin I (left) and insulin II (right). Maps were calculated from models without sulfur atoms (see text). The figures were created using PYMOL (DeLano, 2002).

damage is also limited to only the exposed volume. Moreover, since the recovery of $R_{\text {int }}$ for data set fresh of thaumatin I was not complete (see Fig. $5 c$ ), there seems to be good correspondence between the non-specific damage as observed in the form of an increase of the internal $R$-factor and the specific changes of the electron density.

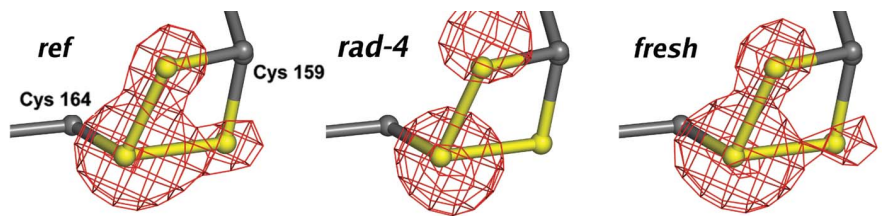

Figure 7

$F_{o}-F_{c}$ difference Fourier maps of the disulfide bond Cys159-Cys164 of thaumatin I for data sets ref, rad-4 and fresh contoured at 3.5 $\sigma$. Maps were calculated from models without sulfur atoms (see text). The figures were created using PYMOL (DeLano, 2002).

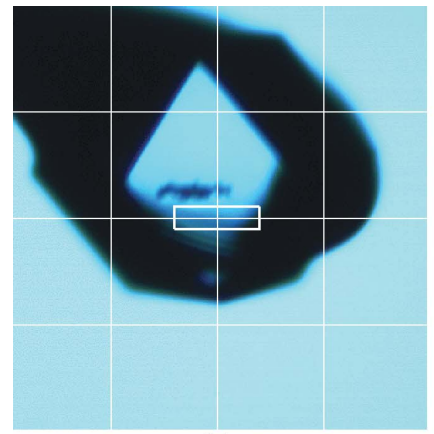

(a)

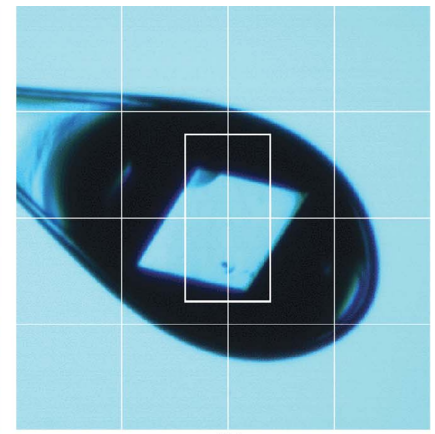

(b)
Figure 8

Microscope images of $(a)$ insulin I and $(b)$ insulin II after the crystals were warmed to $180 \mathrm{~K}$ at the end of the experiment. The white box indicates the beam dimensions, and the dark patch on the insulin I crystal shows the most damaged area, corresponding to the centre of rotation during the experiment. The area is no longer in the box as, on warming, the crystal moved into the cryobuffer. For the insulin II crystal, where the beam was vertically defocused, there is no visible damage.

Fig. 8 shows photographs of insulin crystals I and II after they were warmed up through the glass transition temperature at around $155 \mathrm{~K}$ (Weik et al., 2001) to $180 \mathrm{~K}$ using the cryostat. While there is no indication of a beam footprint for the defocused beam, the left-hand image clearly exhibits a dark line of the size of the beam, which appeared at temperatures above $170 \mathrm{~K}$. The data sets heat- 1 and heat- 2 taken in the fresh wedge and the highly irradiated wedge, respectively, both for the focused and defocused beam, show that the crystal quality is significantly better in the fresh zone of the crystal exposed to the focused beam, whereas both wedges of the crystal exposed to the defocused beam yield equally poor $R$-factors and $I / \sigma_{I}$ ratios (insulin I, heat-1: $R_{\text {int }} 5.9 \%$; heat- $2: R_{\text {int }} 8.8 \%$; insulin II: $R_{\text {int }} 5.9 \%$; heat- $\left.2: R_{\text {int }} 6.6 \%\right)$.

\section{Conclusions}

While the whole sample volume is irradiated at lower dose rate in the case of the vertically defocused beam, in the case of the focused beam, fresh crystal material is moved into the beam owing to the sample rotation. Even when the centre of the crystal receives a dose several times in excess of the Henderson limit, the crystal still diffracts with the initial (insulin) or close to the initial (thaumatin) quality when a previously un-irradiated angular range is exposed. The lessperfect recovery in the case of thaumatin may have its origin in 
the fact that the cross section of the crystal was almost two times smaller than that of the insulin crystal, resulting in a less pronounced distribution of the dose when the crystal is rotated. Our results allow us to conclude that the radiation damage is confined to the irradiated volume, which can be explained by the short range of the photoelectrons (typically a few hundred $\AA$ ) at crystallographic wavelengths and the immobilization of free radicals below the glass transition temperature. For crystals whose dimensions exceed that of the beam, the dose as calculated for a stationary crystal is not a good metric for the estimation of radiation damage: its rotation in the beam must be taken into account. In addition, thermal gradients that may arise between exposed and unexposed parts of the crystal do not cause a measurable deterioration of the crystal. This finding is in good agreement with results from computational fluid dynamics studies conducted by Mhaisekar et al. (2005), which show that thermal gradients within a crystal are extremely small owing to the very high thermal conductivity of protein crystals. The increase of the internal $R$-factor and the decrease of $I / \sigma_{I}$ are reliable indicators of the deterioration of the electron density, e.g. in the form of breaking of disulfide bridges. On the other hand, the rate of the unit-cell expansion seems to vary significantly for crystals of the same protein. Crystal mosaicity should be considered with caution because crystal anisotropy may mask changes due to radiation damage. Integration and scaling of the data sets should therefore follow data acquisition immediately, in order to detect changes of $I / \sigma_{I}$ or of the internal $R$-factor at high resolution as early as possible.

In view of the higher resolution that can be achieved in particular for weakly diffracting crystals by using a small beam size, the use of a vertically focused beam is recommended, even when the crystal is larger than the vertical focus size. The consequences of the enhanced dose rate are mitigated in this case by the rotation of the crystal and can be further reduced by collecting the data set in angularly separated wedges. The latter mode offers the additional advantage of allowing the data completeness to be maximized (Dauter, 1999).

The authors wish to express their gratitude to the referees and the editor for very valuable comments during the reviewing process. AW acknowledges financial support of the NCCR Structural Biology.

\section{References}

Blake, C. C. F. \& Phillips, D. C. (1962). Proceedings of the Symposium on the Biological Effects of Ionizing Radiation at the Molecular Level, Brno, IAEA Symposium, Vienna, pp. 183-191.

Burmeister, W. P. (2000). Acta Cryst. D56, 328-341.

Dauter, Z. (1999). Acta Cryst. D55, 1703-1717.

DeLano, W. L. (2002). The PyMOL Molecular Graphics System. DeLano Scientific, San Carlos, CA, USA.

Garman, E. F. \& Nave, C. (2002). J. Synchrotron Rad. 9, 327.

Henderson, R. (1990). Proc. R. Soc. London, B241, 6-8.

Kabsch, W. (1993). J. Appl. Cryst. 26, 795-800.

Mhaisekar, A., Kazmierczak, M. J. \& Banerjee, R. (2005). J. Synchrotron Rad. 12, 318-328.

Murray, J. W. \& Garman, E. F. (2002). J. Synchrotron Rad. 9, 347-354.

Murray, J. W., Garman, E. F. \& Ravelli, R. B. G. (2004). J. Appl. Cryst. 37, 513-522.

O'Neill, P., Stevens, D. L. \& Garman, E. F. (2002). J. Synchrotron Rad. 9, 329-332.

Ravelli, R. B. G. \& McSweeney, S. (2000). Structure, 8, 315-328.

Ravelli, R. B. G., Theveneau, P., McSweeney, S. \& Caffrey, M. (2002). J. Synchrotron Rad. 9, 355.

Rossetti, D., Lienert, U., Pradervand, C., Schneider, R., Shi, M., Zelenika, S., Rossat, M., Hignette, O., Rommeveaux, A. \& SchulzeBriese, C. (2002). Proc. SPIE, 4782, 86-93.

Sheldrick, G. M. \& Schneider, T. R. (1997). Methods Enzymol. 277, 319-341.

Teng, T. Y. \& Moffat, K. (2000). J. Synchrotron Rad. 7, 313-317.

Weik, M., Kryger, G., Schreurs, A. M. M., Bouma, B., Silman, I., Sussman, J. L., Gros, P. \& Kroon, J. (2001). Acta Cryst. D57, 566573.

Wikoff, W. R., Schildkamp, W. \& Johnson, J. E. (2000). Acta Cryst. D56, 890-893. 